\title{
PROPOSTA METODOLÓGICA PARA AVALIAÇÃO DE CONTROLADOR AUTOMÁTICO DE SEÇÕES E PULVERIZAÇÃ̃O ${ }^{1}$
}

\author{
ÉTORE F. REYNALDO ${ }^{2}$, JOSÉ P. MOLIN ${ }^{3}$
}

\begin{abstract}
RESUMO: Na aplicação de produtos fitossanitários, a utilização de equipamentos que controlam automaticamente as seções da barra e a pulverização já é realidade; entretanto, ainda não há resultados que demonstrem a sua eficácia. Por esse motivo, este trabalho teve por finalidade desenvolver uma metodologia para a avaliação de um equipamento que controla automaticamente as seções e a pulverização. Avaliou-se um controlador automático de seções e pulverização de mercado, e, para tanto, foram utilizados três níveis de acurácia do sinal de GPS (algoritmo interno, SBAS e RTK), três ângulos para a simulação de entrada e saída da barra de pulverização em relação à borda do talhão $\left(0 ; 45 \mathrm{e} 60^{\circ}\right)$ e três velocidades de trabalho $\left(1,66 ; 5,00\right.$ e $\left.8,33 \mathrm{~m} \mathrm{~s}^{-1}\right)$. A metodologia proposta possibilitou a determinação dos tempos e distâncias de abertura e fechamento das seções. Os coeficientes de variação para os tempos e distâncias de abertura e fechamento das seções indicaram uma variação considerável. Houve interações significativas em função do tipo de sinal de GPS. A configuração recomendada pelo fabricante e adotada para a avaliação do controlador automático de seções e pulverização não atende a todas as situações simuladas.
\end{abstract}

PALAVRAS-CHAVE: agricultura de precisão, aplicação de produtos fitossanitários, sistemas eletrônicos.

\section{PROPOSED METHODOLOGY FOR EVALUATION OF AUTOMATIC CONTROLLER OF BOOM SECTIONS AND SPRAY}

\begin{abstract}
In the application of pesticides, the use of equipment that automatically controls the sections of the boom and the spray is already reality. However, there are no results to show its effectiveness. Therefore, this work was to develop a methodology for the evaluation of equipments which automatically controls the boom sections and the spraying pattern. A commercial automatic controller of boom spraying sections was evaluated using three levels of GPS accuracy (internal algorithm, SBAS and RTK), three angles for the simulation of entry and exit of the spray bar on field edges $\left(0,45\right.$ and $\left.60^{\circ}\right)$ and three forward speeds $\left(1.66,5.00\right.$ and $\left.8.33 \mathrm{~m} \mathrm{~s}^{-1}\right)$. The proposed methodology allowed the determination of opening and closing times of the electric valves. The coefficients of variation indicated a considerable variation. There were significant interactions depending on the type of GPS signal used. The manufacturer recommended configuration, used for the evaluation of the automatic boom sections and spraying controller, does not attended all the simulated situations.
\end{abstract}

KEYWORDS: precision agriculture; application of pesticides; electronic systems.

\section{INTRODUÇÃO}

Durante todo o ciclo de produção de matérias-primas destinadas a alimentos, fibras e energia, a aplicação dos produtos fitossanitários é um dos pontos críticos. Os defensivos químicos representam uma das principais formas de se combaterem as pragas, doenças e plantas daninhas das culturas. Entretanto, a tecnologia de aplicação de produtos fitossanitários continua sendo um dos pontos de estrangulamento na produção agrícola, pois se necessita melhorar a eficiência nas

\footnotetext{
${ }^{1}$ Extraído da dissertação de mestrado do primeiro autor.

${ }^{2}$ Eng $^{\circ}$ Agrônomo, Doutorando, Programa DE Pós-Graduação em Energia na Agricultura, Faculdade de Ciências Agronômicas, UNESP, etfreynaldo@yahoo.com.br.

${ }^{3}$ Professor Associado, Departamento de Engenharia de Biossistemas, ESALQ/USP, Piracicaba - SP, jpmolin@ usp.br. 
aplicações em lavouras, diminuindo gastos e desperdícios, aumentando assim o retorno econômico e evitando contaminações ambientais.

Uma solução para a melhoria da baixa eficiência nas aplicações de produtos fitossanitários, de acordo com BATTE \& EHSANI (2006), seria a utilização de sistemas que controlam automaticamente as seções e a pulverização, a qual pode reduzir significativamente a sobreposição, poupando produtos fitossanitários, combustível e o tempo durante o processo de aplicação, resultando em maior rendimento operacional e também evitando as falhas entre as passadas. Além disso, ajuda a reduzir os esforços mentais do operador, diminuindo sua fadiga e aumentando seu rendimento e qualidade de trabalho.

O uso dos sistemas que controlam automaticamente as seções e a pulverização deu-se graças à evolução da computação e das tecnologias de comunicação, em que sensores e atuadores são incorporados nas máquinas agrícolas ou em aeronaves, a fim de fornecer informações úteis sobre as heterogeneidades das culturas e da presença de plantas daninhas, por exemplo. Atenção especial pode ser dada à redução de herbicidas utilizados, que são os principais poluentes na agricultura (BOSSU et al., 2008). Segundo ZAMBOLIM et al. (2008), nos tratamentos fitossanitários com o emprego de herbicidas, a aplicação localizada e em taxa variável pode significar redução de mais de $60 \%$ nas quantidades de produtos fitossanitários utilizados. O uso de tal tecnologia remete para o campo de estudos denominado de Agricultura de Precisão (AP) (McBRATNEY et. al, 2005). A AP surge com a promessa de reverter a situação atual, possibilitando a aplicação de insumos agrícolas de forma localizada e somente na quantidade necessária (LIMA et. al, 2006). Entretanto, de acordo com SCHULLER (1991), o tempo de resposta desses equipamentos é um dos fatores-chave para o desempenho das máquinas de aplicação dotadas de controle eletrônico, principalmente em sistemas de aplicação localizada de defensivos químicos.

Assim, o presente trabalho tem por finalidade desenvolver critérios e procedimentos para avaliar equipamentos que controlam automaticamente a abertura e $\mathrm{O}$ fechamento das sessões de pulverizadores de barra. Como o desempenho desse tipo de dispositivo é dependente de fatores como a qualidade do sinal dos sistemas GNSS, formato do contorno das lavouras expressos pelo ângulo de entrada e saída da barra pulverizadora em relação à borda do talhão e à velocidade de operação, estes foram utilizados para avaliar o tempo para o início e término da pulverização em relação à linha delimitadora (borda do talhão) de um equipamento comercial que controla automaticamente as seções e a pulverização.

\section{MATERIAL E MÉTODOS}

A avaliação do controlador automático de seção e pulverização foi realizada nas dependências do Centro de Pesquisa \& Desenvolvimento da empresa Máquinas Agrícolas Jacto S.A., situadas nas coordenadas geográficas $22^{\circ} 06^{\prime} 39^{\prime}$ 'S e 50¹5’08”W, no município de Pompeia - SP.

O equipamento submetido à avaliação foi o controlador automático de seções e pulverização

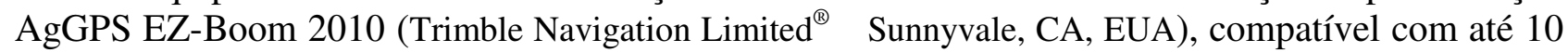
seções. O sistema de controle automático de seções da barra de pulverização trabalha em conjunto com o sistema de orientação por barra de luzes AgGPS EZ-Guide 500 (Trimble Navigation Limited $\square$ Sunnyvale, CA, EUA), utilizado como tela de configuração para o controlador. O equipamento foi montado em um pulverizador autopropelido Uniport Star 2500 (Máquinas Agrícolas Jacto, Pompeia - SP), com 24 m de barra, divididas em oito seções. Na Figura 1, é apresentado o esquema de montagem do controlador.

Para a determinação da velocidade de deslocamento durante os percursos do veículo (pulverizador autopropelido), foi utilizado um sensor de radar de velocidade Mid-Tech RGSS201(Midwest Technologies, Springfield, IL, EUA). Para a determinação do tempo de início e término da pulverização, ou seja, o fluxo de água nas pontas de pulverização, em relação à borda do talhão, foram utilizados dois transdutores elétricos de pressão Wika C-10 (Wika Instrument Corporation, Lawrenceville, GA, EUA), com faixa de trabalho 0 a 2,41 MPa, acoplados aos últimos 
bicos de pulverização, nas extremidades da barra do pulverizador. Para a determinação da posição da borda do talhão, ou seja, o ponto exato de entrada ou saída da barra de pulverização, foram utilizados dois sensores fotodiodos OR6K-30GI70-A2 (Sense Sensores e Instrumentos, São PauloSP), com tempo de resposta de $20 \mathrm{~m} \mathrm{~s}^{-1}$, fixados nas extremidades das barras de pulverização. Utilizou-se um coletor de dados ADS-2000 (Lynx Tecnologia Eletrônica Ltda., São Paulo - SP), com frequência de coleta de $200 \mathrm{~Hz}$, e os dados foram armazenados em um computador portátil.

Para a demarcação da pista de ensaio com os diferentes ângulos, foi utilizado um teodolito topográfico óptico mecânico.

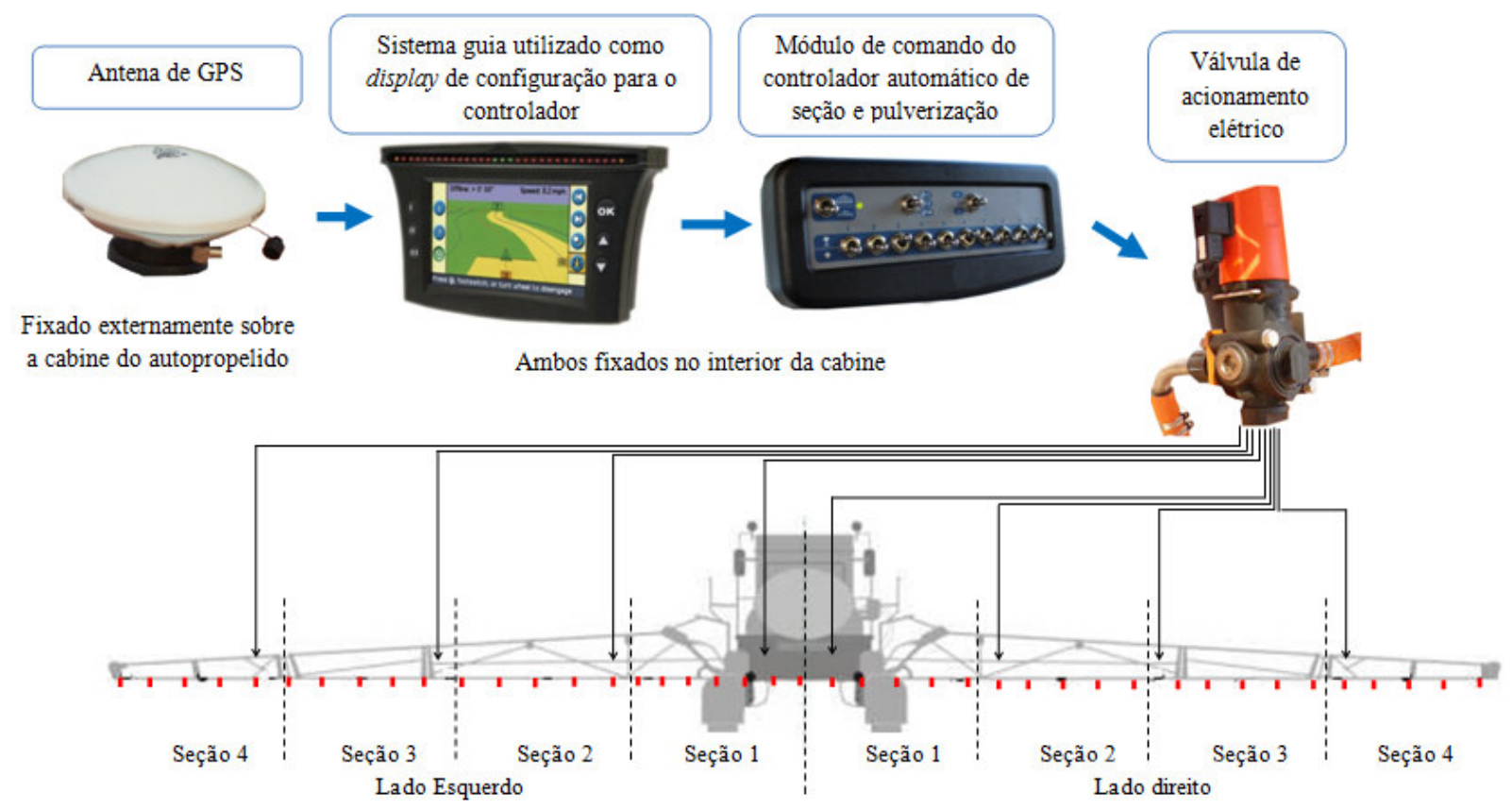

FIGURA 1. Esquema de montagem do controlador automático de seções e pulverização, suas respectivas partes e posições. Setup of the automatic controller of boom sections, respective parts and positions.

As variáveis estabelecidas para a avaliação do controlador foram: sinal de GPS, velocidades de deslocamento do veículo e ângulos de simulação para entrada e saída da barra de pulverização em relação à borda do talhão. Os sinais avaliados foram um sistema RTK, um sinal de correção comercial SBAS ("satellite-based augmentation system", ou via satélite) VBS (OmniStar, Houston, TX, EUA) e um receptor de GPS L1, do próprio fabricante do controlador, apenas com o recurso de algoritmo interno de filtragem de erros de posicionamento ("firmware"), comum no mercado brasileiro para aplicações em sistema de guia por barra de luzes.

Para associar a qualidade de posicionamento com o tempo de resposta do sistema de controle, foram selecionadas três velocidades de deslocamento para o veículo. Utilizaram-se como metas as velocidades de 1,$66 ; 5,00$ e $8,33 \mathrm{~m} \mathrm{~s}^{-1}\left(6 ; 18\right.$ e $\left.30 \mathrm{~km} \mathrm{~h}^{-1}\right)$.

Para avaliar especialmente a geometria dos talhões e dos obstáculos internos a estes, onde o sistema deve atuar controlando automaticamente o início e o término da pulverização nas seções da barra de pulverização, visando a minimizar a sobreposição e as falhas na pulverização, foram adotados três ângulos para a simulação de entrada e saída da barra de pulverização do pulverizador em relação à borda do talhão, sendo eles: 0; 45 e 60. Na Figura 2, é apresentado o croqui da pista de teste com seus respectivos ângulos demarcados em solo e georreferenciados com sistema GPS RTK. 


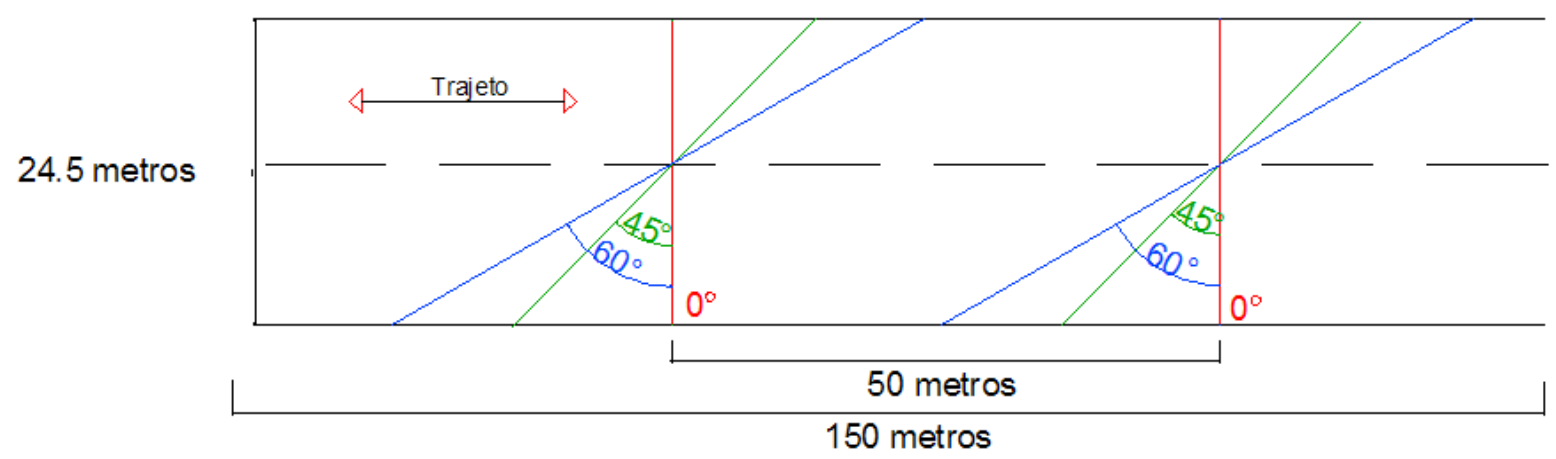

FIGURA 2. Ângulos de simulação para entrada e saída da barra de pulverização em relação à borda do talhão: ângulo de $0^{\circ}$ (vermelho); ângulo de $45^{\circ}$ (verde) e ângulo de $60^{\circ}$ (azul). Entry and exit simulated angles of the spray boom on the field edges: (red) $0^{\circ}$ angle, (green) $45^{\circ}$ angle and (blue) $60^{\circ}$ angle.

Para determinar o tempo de resposta do controlador em mudar a posição das válvulas de acionamento elétrico (abrir ou fechar), decorrendo o início ou o término da pulverização em função da linha delimitadora da borda do talhão, aplicou-se a eq.(1), para o caso com tratamentos com ângulo de $0^{\circ}$. Se o valor encontrado é negativo (-), significa que a válvula de acionamento elétrico abriu antes que o pulverizador ultrapassasse a borda do talhão, e se o valor é positivo (+), significa que ele abriu após ultrapassar a linha delimitadora da borda do talhão.

$$
\mathrm{T}_{\mathrm{a}}\left(\text { ou } \mathrm{T}_{\mathrm{f}}\right)=\mathrm{T}_{\mathrm{va}}-\mathrm{T}_{\mathrm{fd}}
$$

em que,

$\mathrm{T}_{\mathrm{a}}$ - tempo de abertura, $\mathrm{s}$

$\mathrm{T}_{\mathrm{f}}$ - tempo de fechamento, $\mathrm{s}$;

$\mathrm{T}_{\mathrm{va}}$ - tempo da válvula, $\mathrm{s}, \mathrm{e}$

$\mathrm{T}_{\mathrm{fd}}$ - tempo do fotodiodo, $\mathrm{s}$.

Os tempos para a abertura e/ou o fechamento das válvulas de acionamento elétrico, bem como o início ou o término da pulverização para os tratamentos que envolviam os ângulos de $45^{\circ}$ e $60^{\circ}$, foram determinados geometricamente, a partir de um ponto de origem $\left(\mathrm{P}_{\mathrm{o}}\right)$. Com o auxílio de um software CAD (Desenho Assistido por Computador), determinaram-se as distâncias para a abertura ou o fechamento das válvulas para cada seção, em função da linha delimitadora da borda do talhão e de acordo com o ângulo utilizado.

$\mathrm{O}$ delineamento do ensaio, para a validação da metodologia e avaliação do controlador automático de seções e pulverização, compreendeu um experimento com arranjo fatorial 3x3x3, com quatro repetições. As análises estatísticas foram realizadas utilizando o programa SAS (1998). Primeiramente, aplicou-se a análise de homogeneidade dos dados e, posteriormente, procedeu-se à remoção dos dados anormais existentes. A comparação da interação tripla dos tratamentos e da relação entre as variáveis foi avaliada por meio de análise de variância, verificando-se a significância, pelo teste $\mathrm{F}$ de Snedecor, a $5 \%$ de significância. Para os tratamentos significativos, aplicou-se o desmembramento em matriz, pelo teste Tukey, a 5\% de significância, em função da variável sinal de GPS.

\section{RESULTADOS E DISCUSSÃO}

Nas Figuras 3; 4 e 5, têm-se os tempos e as distâncias para o início e o término da pulverização para as seções extremas (seção 4) esquerda e direita, com ângulo de 0; 45 e $60^{\circ}$, respectivamente, para a simulação de entrada e saída da barra de pulverização em relação à borda do talhão, utilizando o sinal de GPS com algoritmo interno (firmware) (gráficos a, b), VBS (c, d) e sistema RTK ( e, f), nas três velocidades. 
Nota-se que, para a menor velocidade $\left(1,66 \mathrm{~m} \mathrm{~s}^{-1}\right)$, utilizando o sinal de GPS com o algoritmo interno para a correção dos erros de posicionamento e ângulo de $0^{\circ}$ para a simulação de entrada e saída da barra de pulverização (Figura 3a), o deslocamento da reação de abertura das seções do pulverizador diferenciou-se dos demais. Isso decorreu do desvio no ponto de início da pulverização, ocorrendo 0,52 s ou $0,93 \mathrm{~m}$ após a barra do pulverizador ter cruzado a linha delimitadora da borda do talhão.

Nos demais tratamentos, com a utilização do sinal VBS e sistema RTK, não foram identificados desvios para o ponto de abertura do fluxo hidráulico (falha na aplicação). Entretanto, foram observadas grandes distâncias para a abertura das seções da barra do pulverizador em relação à borda do talhão, para as velocidades 2 e 3, com valores de $-2,63$ a $-5,61 \mathrm{~m}$ para o uso do sinal VBS e de $-3,79$ a $-5,68 \mathrm{~m}$ com o uso do sistema RTK, indicando um desperdício de produto em função de a abertura da pulverização dar-se distante da borda do talhão.

Os coeficientes de variação (C.V.), calculados para os tempos de início e término da pulverização (Tabela 1), indicam, além de uma variação entre as diferentes repetições, também um comportamento distinto entre os lados das seções analisadas da barra do pulverizador. É fato que os maiores valores de C.V. se apresentam mais expressivos nos tratamentos que envolvem os ângulos de 45 e $60^{\circ}$ para a simulação de entrada e saída do talhão. Vários fatores podem contribuir para esta variação, pois ao se analisarem o início e o término da pulverização, têm-se a interação de diversos componentes e, em especial, o circuito hidráulico e seus componentes eletroeletrônicos (válvulas e controladores).

(a)

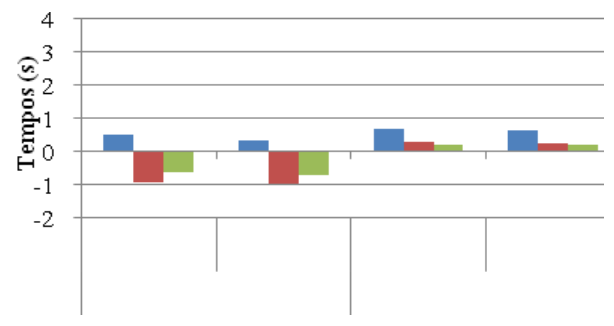

(c)

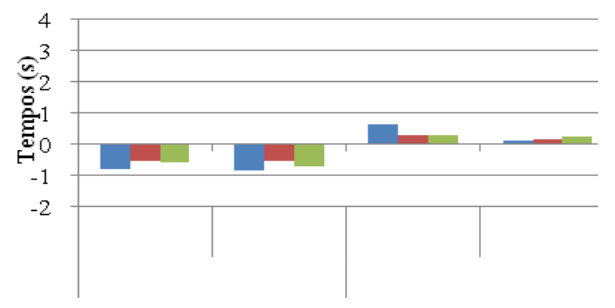

(e)

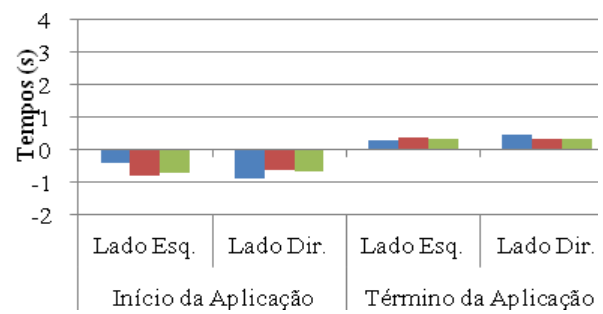

Velocidade $1,66\left(\mathrm{~m} \mathrm{~s}^{-1}\right)$ (b)

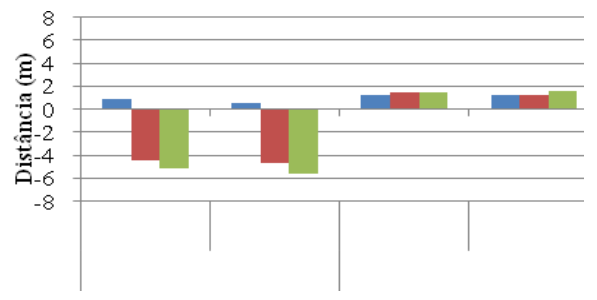

(d)

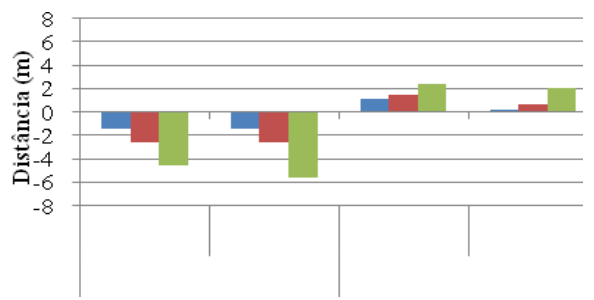

(f)

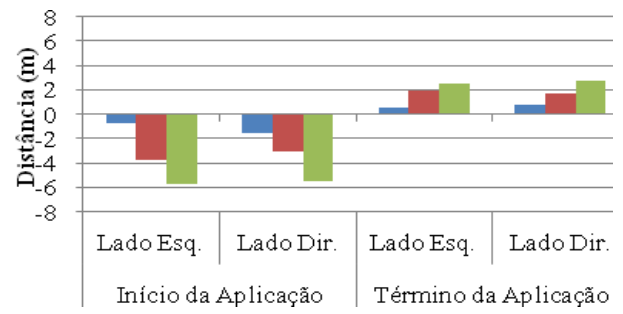

FIGURA 3. Tempos e distâncias de início ou término da aplicação nas seções extremas da barra de pulverização (4 $4^{\mathrm{a}}$ esquerda e direita), com ângulo de $0^{\circ}$ para a simulação de entrada e saída da barra em relação à borda, utilizando os sinais de GPS com algoritmo interno (firmware) (a, b), VBS (c, d) e sistema RTK (e, f), nas três velocidades. Time and distance of the beginning or the end of the application at the extreme boom sections ( $4^{\text {th }}$ left and right), at $0^{\circ}$ of simulated entry and exit of the bar on the edges of the field, using the internal algorithm (firmware) (a , b), VBS (c, d) and RTK system (e, f) in three speeds. 
Para os tempos e distâncias de início e término da pulverização, com ângulo de $45^{\circ}$ (Figura 4), não foram observados desvios nos pontos de início ou término da aplicação em relação à borda do talhão. Porém, como já observado para os tempos e distâncias de início e término da pulverização, com ângulo de $0^{\circ}$, apresentam grandes distâncias de início ou término da pulverização em relação à borda do talhão.

(a)

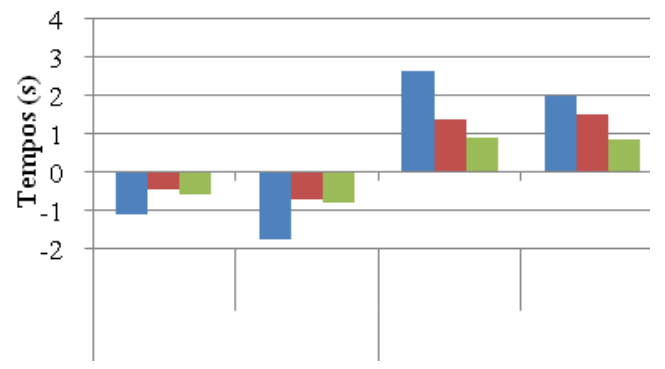

(c)

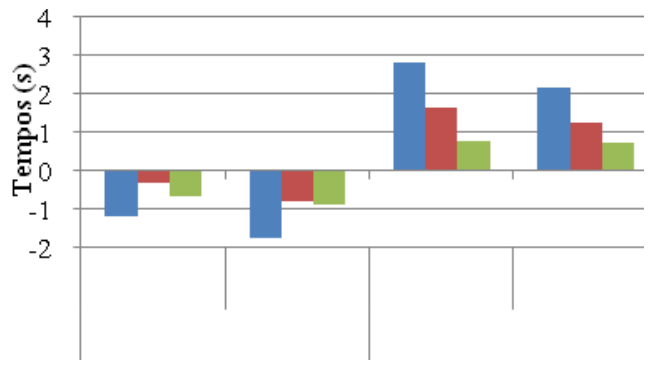

(e)

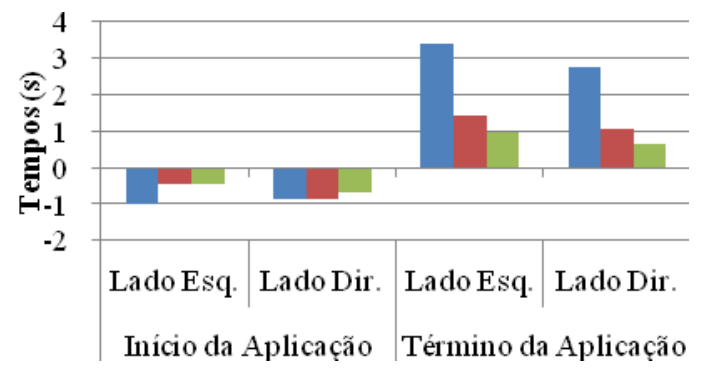

(b)

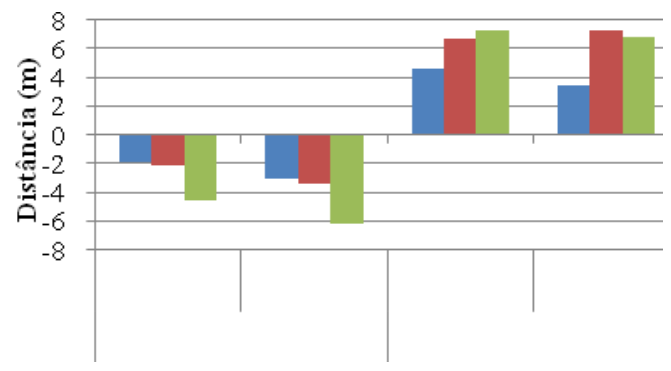

(d)

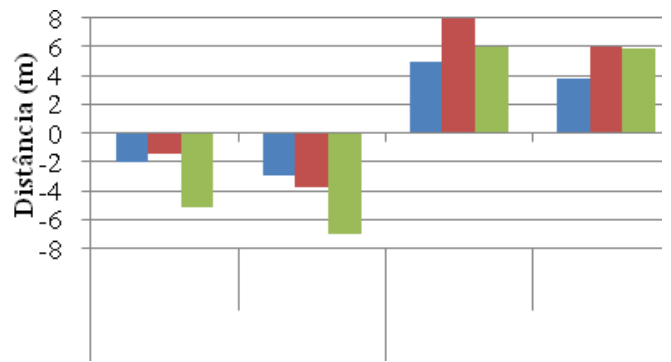

(f)

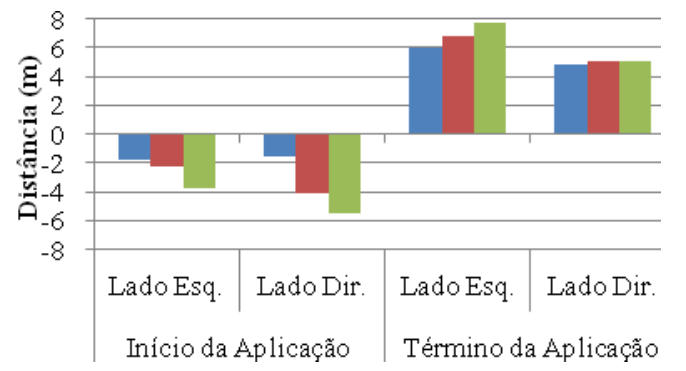

Velocidade $1,66\left(\mathrm{~m} \mathrm{~s}^{-1}\right)$

Velocidade 5,00 $\left(\mathrm{m} \mathrm{s}^{-1}\right)$

Velocidade $8,33\left(\mathrm{~m} \mathrm{~s}^{-1}\right)$

FIGURA 4. Tempos e distâncias de início ou término da aplicação nas seções extremas da barra de pulverização (4 $4^{\mathrm{a}}$ esquerda e direita), com ângulo de $45^{\circ}$ para a simulação de entrada e saída da barra em relação à borda, utilizando o algoritmo interno (firmware) (a, b), VBS (c, d) e sistema RTK (e, f), nas três velocidades. Time and distance of the beginning or the end of the application at the extreme boom sections $\left(4^{\text {th }}\right.$ left and right), at $45^{\circ}$ of simulated entry and exit of the bar on the edges of the field, using the internal algorithm (firmware) (a , b), VBS (c, d) and RTK system $(e, f)$ in three speeds.

Aparentemente, a velocidade tem maior influência no comportamento dos tempos de início ou término da pulverização que o sinal de GPS utilizado, como pode ser observado na Figura 4 (a, c, e), para as três velocidades, mais expressivos nos tempos de término da pulverização. Este fato é relatado no guia do manual do usuário do controlador automático de seção e pulverização avaliado.

O fabricante relata que o algoritmo do controlador não leva em consideração a velocidade de deslocamento para os cálculos, a fim de se determinar o tempo correto para o fechamento das válvulas de acionamento elétrico de cada seção da barra do pulverizador, resultando em atrasos crescentes nos tempos de término de pulverização entre as diferentes velocidades avaliadas. 
(a)

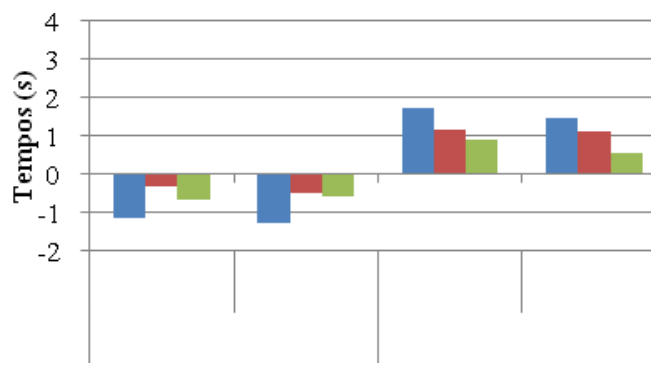

(c)

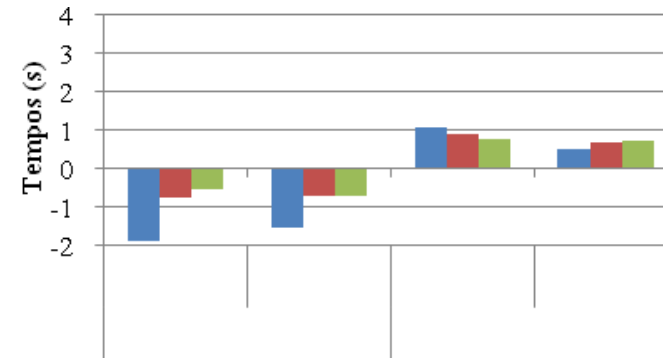

(e)

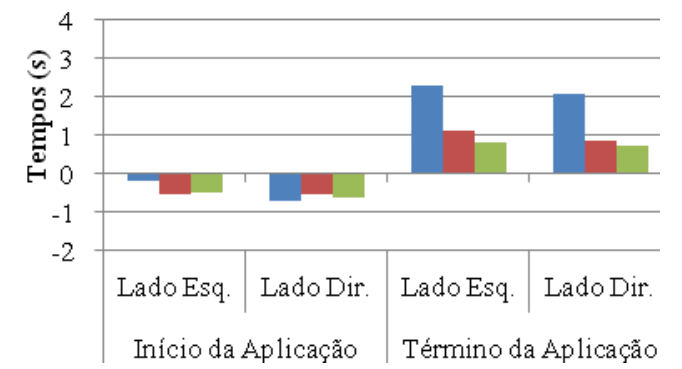

Velocidade $1,66\left(\mathrm{~m} \mathrm{~s}^{-1}\right)$ (b)

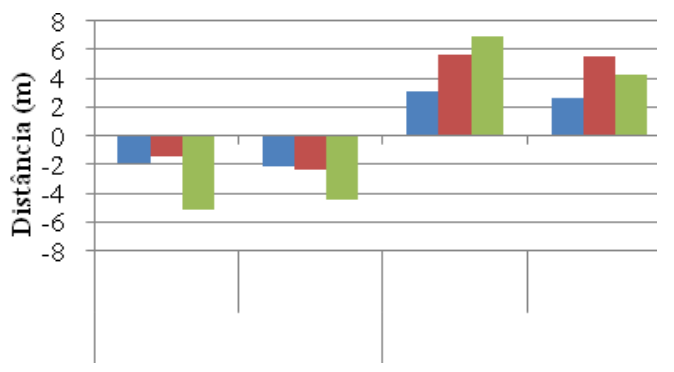

(d)

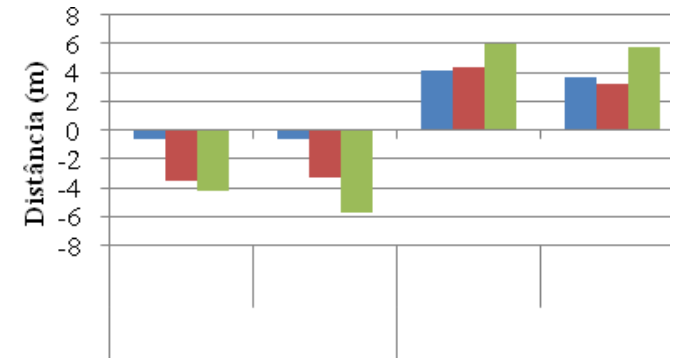

(f)

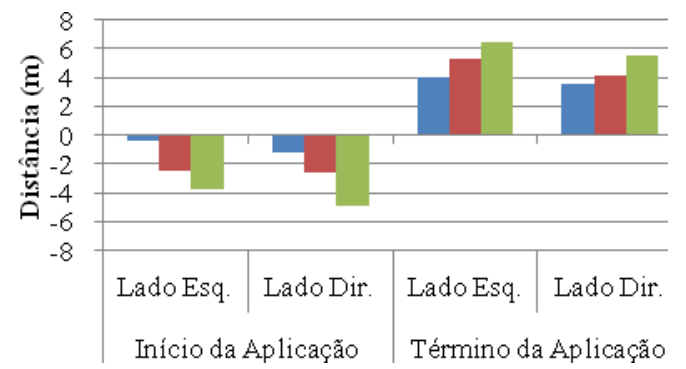

Velocidade 8,33 ( $\left.\mathrm{m} \mathrm{s}^{-1}\right)$

FIGURA 5. Tempos e distâncias de início ou término da aplicação nas seções da barra de pulverização (4⿳亠丷a esquerda e direita), com ângulo de $60^{\circ}$ para a simulação de entrada e saída da barra em relação à borda, utilizando o algoritmo interno (firmware) (a, b), VBS (c, d) e sistema RTK (e, f), nas três velocidades. Time and distance of the beginning or the end of the application at the extreme boom sections ( $4^{\text {th }}$ left and right), at $60^{\circ}$ of simulated entry and exit of the bar on the edges of the field, using the internal algorithm (firmware) $(a, b)$, VBS (c, d) and RTK system (e, f) in three speeds.

Contudo, quando convertidos esses tempos em distâncias, a velocidade de $8,33 \mathrm{~m} \mathrm{~s}^{-1}$ destaca-se em relação às demais, como pode ser visto na Figura 5. Os maiores coeficientes de variação das distâncias de término da pulverização foram observados para os tratamentos que utilizaram o ângulo de $60^{\circ}$ para a entrada e saída da barra de pulverização, em relação à borda do talhão (Tabela $1)$.

Nota-se que os valores de C.V. foram maiores quando simuladas a entrada e a saída da barra de pulverização em ângulos de 60 e $45^{\circ}$.

Todas as distâncias de início ou término da pulverização, independentemente dos sinais de GPS, velocidades e ângulos utilizados, foram maiores que as distâncias assumidas por BATTE \& EHSANI (2006). Estes realizaram simulações de uso de um controlador de seções complementado por sistema-guia de atuador de volante no pulverizador autopropelido e consideraram a utilização de um sistema RTK para posicionamento em campo, com uma distância de 0,05 $\mathrm{m}$ de sobreposição, tanto para a abertura quanto para o fechamento das seções. 
TABELA 1. Coeficiente de variação para as distâncias $(m)$ de início e término da pulverização em simulação de entrada e saída no talhão, variando sinal de GPS, ângulos e velocidade de deslocamento. Coefficients of variation (\%) for the distance $(m)$ of the beginning and the end of the spraying times (sec.) for the forward speeds, GPS signals and entrance (opening) and exit (closing) field angles for both boom sides.

\begin{tabular}{|c|c|c|c|c|c|c|}
\hline \multirow{3}{*}{$\begin{array}{l}\text { Sinal de } \\
\text { GPS }\end{array}$} & \multirow{3}{*}{$\begin{array}{l}\text { Velocidade } \\
\left(\mathrm{m} \mathrm{s}^{-1}\right)\end{array}$} & \multirow{3}{*}{ Ângulo } & \multicolumn{4}{|c|}{ Coeficiente de Variação (\%) } \\
\hline & & & \multicolumn{2}{|c|}{ Início da Pulverização (m) } & \multicolumn{2}{|c|}{ Término da Pulverização (m) } \\
\hline & & & Lado Esquerdo & Lado Direito & Lado Esquerdo & Lado Direito \\
\hline \multirow{9}{*}{ Firmware } & \multirow{3}{*}{1,6} & $0^{\circ}$ & 14,1 & 18,8 & 53,3 & 57,9 \\
\hline & & $45^{\circ}$ & 39,3 & 48,2 & 99,1 & 79,7 \\
\hline & & $60^{\circ}$ & 44,0 & 61,6 & 90,3 & 109,3 \\
\hline & \multirow{3}{*}{5,0} & $0^{\circ}$ & 34,5 & 29,2 & 107,8 & 109,7 \\
\hline & & $45^{\circ}$ & 106,9 & 131,8 & 95,3 & 94,8 \\
\hline & & $60^{\circ}$ & 100,3 & 68,9 & 74,2 & 74,9 \\
\hline & \multirow{3}{*}{8,3} & $0^{\circ}$ & 20,8 & 8,6 & 87,3 & 93,3 \\
\hline & & $45^{\circ}$ & 50,6 & 64,0 & 55,6 & 75,3 \\
\hline & & $60^{\circ}$ & 30,9 & 15,7 & 60,7 & 40,6 \\
\hline \multirow{9}{*}{ VBS } & \multirow{3}{*}{1,6} & $0^{\circ}$ & 39,6 & 37,6 & 95,3 & 149,3 \\
\hline & & $45^{\circ}$ & 99,1 & 120,6 & 49,4 & 53,4 \\
\hline & & $60^{\circ}$ & 77,6 & 52,2 & 36,0 & 47,1 \\
\hline & \multirow{3}{*}{5,0} & $0^{\circ}$ & 34,1 & 22,5 & 49,5 & 66,7 \\
\hline & & $45^{\circ}$ & 50,8 & 43,2 & 28,0 & 65,2 \\
\hline & & $60^{\circ}$ & 75,8 & 29,3 & 33,9 & 15,2 \\
\hline & \multirow{3}{*}{8,3} & $0^{\circ}$ & 33,9 & 40,6 & 6,8 & 14,3 \\
\hline & & $45^{\circ}$ & 37,8 & 32,1 & 7,3 & 25,9 \\
\hline & & $60^{\circ}$ & 54,8 & 41,3 & 17,6 & 19,3 \\
\hline \multirow{9}{*}{ RTK } & \multirow{3}{*}{1,6} & $0^{\circ}$ & 55,7 & 7,0 & 91,6 & 113,9 \\
\hline & & $45^{\circ}$ & 36,9 & 21,6 & 38,2 & 37,5 \\
\hline & & $60^{\circ}$ & 72,4 & 22,2 & 49,5 & 31,0 \\
\hline & \multirow{3}{*}{5,0} & $0^{\circ}$ & 25,1 & 21,5 & 51,1 & 57,8 \\
\hline & & $45^{\circ}$ & 29,9 & 17,0 & 33,7 & 49,5 \\
\hline & & $60^{\circ}$ & 93,4 & 32,0 & 89,8 & 48,9 \\
\hline & \multirow{3}{*}{8,3} & $0^{\circ}$ & 21,9 & 20,6 & 3,7 & 20,0 \\
\hline & & $45^{\circ}$ & 23,7 & 29,9 & 5,3 & 10,2 \\
\hline & & $60^{\circ}$ & 67,7 & 29,7 & 17,2 & 31,6 \\
\hline
\end{tabular}

Foram identificadas interações significativas entre os diferentes sinais de GPS, ângulos e velocidades para os tempos de início de pulverização, a 5\% de significância, pelo teste de Tukey (Tabela 2).

$\mathrm{Na}$ Tabela 3, são apresentados os tratamentos com os valores dos tempos (s) significativos após desmembramento pela matriz de Tukey, onde foi possível estabelecer-se as correlações entre os diferentes tratamentos. Para a velocidade de $1,66 \mathrm{~m} \mathrm{~s}^{-1}$ e ângulos de 45 e $60^{\circ}$, em início de pulverização, no lado direito da barra do pulverizador, o melhor tratamento foi obtido com o uso do sistema RTK, com médias de $-0,74$, e $-0,89$ s (menores tempos sem falhas). Para o caso dos tratamentos com velocidade de $1,66 \mathrm{~m} \mathrm{~s}^{-1}$, ângulo de $45^{\circ}$ em término de pulverização no lado direito da barra do pulverizador autopropelido, o melhor resultado foi obtido com o uso do sinal de GPS autônomo com o uso do algoritmo interno (firmware).

Os tratamentos que se mostraram significativos para o uso dos diferentes sinais de GPS, concentraram-se na velocidade de $1,66 \mathrm{~m} \mathrm{~s}^{-1}$ e ângulos com inclinação (45 ou 60 $)$ para a entrada ou a saída da barra do pulverizador. Observa-se que aumentando a velocidade de trabalho, dilui-se o 
erro de acurácia dos diferentes sinais de GPS, ou seja, há outros fatores com maior influência nos resultados, como, por exemplo, o ângulo, a velocidade ou mesmo a interação de ambos.

TABELA 2. Tratamentos com interações significativas entre os diferentes sinais de GPS, ângulos e velocidades, para os tempos e distâncias de início e término da pulverização. Treatments with significant interactions between different GPS signals, angles and speeds, for time and distance of the beginning and the end of the spraying.

\begin{tabular}{cccc}
\hline Pulverização & Lado & Tempos (s) & Distâncias (m) \\
\hline \multirow{2}{*}{ Início } & $\begin{array}{c}\text { Esquerdo } \\
\text { Direito }\end{array}$ & GPS x Âng. x Vel. * & $\begin{array}{c}\text { Vel. x Âng. * } \\
\text { n.s }\end{array}$ \\
\hline \multirow{2}{*}{ Término } & Esquerdo & GPS x Âng. x Vel. * & n.s \\
\cline { 2 - 4 } & Direito & GPS x Âng. x Vel. * & n.s \\
\hline
\end{tabular}

n.s - não significativo pelo teste Tukey. * Significativo a 5\% de probabilidade pelo teste $\mathrm{t}$.

TABELA 3. Tratamentos com valores de tempos significativamente distintos após desmembramento em função do sinal GPS utilizado. Treatments with significantly distinct time values after splitting up according to the GPS signals.

\begin{tabular}{|c|c|c|c|c|c|c|}
\hline \multirow{2}{*}{ Operação } & \multirow{2}{*}{ Lado } & \multicolumn{2}{|c|}{ Interação } & \multicolumn{3}{|c|}{ Tempos } \\
\hline & & Sinal de GPS & Velocidade $\left(\mathrm{m} \mathrm{s}^{-1}\right)$ & Ângulo da borda & Média & $\operatorname{Pr}>t$ \\
\hline \multirow{3}{*}{$\begin{array}{c}\text { Início da } \\
\text { pulverização }\end{array}$} & \multirow{3}{*}{ Direito } & $\begin{array}{c}\text { Firmware } \\
\text { VBS }\end{array}$ & 1,66 & $45^{\circ}$ & $\begin{array}{l}-1,26 \\
-1,52\end{array}$ & 0,0219 \\
\hline & & $\begin{array}{c}\text { Firmware } \\
\text { RTK }\end{array}$ & 1,66 & $45^{\circ}$ & $\begin{array}{l}-1,26 \\
-0,71\end{array}$ & 0,0400 \\
\hline & & $\begin{array}{c}\text { Firmware } \\
\text { RTK }\end{array}$ & 1,66 & $60^{\circ}$ & $\begin{array}{l}-1,74 \\
-0,89\end{array}$ & $<0,0001$ \\
\hline \multirow{2}{*}{$\begin{array}{c}\text { Término da } \\
\text { pulverização }\end{array}$} & \multirow{2}{*}{ Direito } & $\begin{array}{c}\text { Firmware } \\
\text { RTK }\end{array}$ & 1,66 & $45^{\circ}$ & $\begin{array}{l}1,48 \\
2,08\end{array}$ & $<0,0001$ \\
\hline & & $\begin{array}{l}\text { VBS } \\
\text { RTK }\end{array}$ & 1,66 & $60^{\circ}$ & $\begin{array}{c}0,5 \\
2,76\end{array}$ & $<0,0001$ \\
\hline
\end{tabular}

$\mathrm{Na}$ Tabela 4, é apresentado o teste de médias para os tratamentos com as distâncias (m) significativas após desmembramento pela matriz de correlação entre as médias dos diferentes tratamentos, não sendo observadas diferenças significativas entre os sinais de GPS VBS e sistema RTK, porém ambos diferem do tratamento que utilizou o sinal de GPS autônomo com algoritmo interno (firmware).

Nota-se que a interação se deu para ângulo $0^{\circ}$ da barra de pulverização em relação à borda. Porém, para os demais tratamentos, não foram identificadas interações significativas em função do sinal de GPS.

TABELA 3. Tratamentos com valores de distâncias significativamente distintas após desmembramento em função do sinal GPS utilizado. Treatments with significantly different distances after splitting up according to the GPS signals.

\begin{tabular}{cccccc}
\hline \multirow{2}{*}{ Operação } & \multirow{2}{*}{ Lado } & \multicolumn{2}{c}{ Interação } & \multicolumn{2}{c}{ Distância $(\mathrm{m})$} \\
\cline { 3 - 6 } & & Sinal de GPS & Ângulo de simulação & Média & Pr $>$ t \\
\hline \multirow{3}{*}{$\begin{array}{c}\text { Início da } \\
\text { pulverização }\end{array}$} & Dirmware & \multirow{2}{*}{$0^{\circ}$} & $-0,95$ & $<0,0001$ \\
& Direito & VBS & & $-0,53$ & $<$ \\
\cline { 3 - 6 } & & Firmware & \multirow{2}{*}{$0^{\circ}$} & $-0,95$ & $<0,0001$ \\
\hline
\end{tabular}


De acordo com STAFFORD et. al (1996), na pulverização e controle da sobreposição, é indicada uma acurácia dos receptores de GPS na ordem de $0,10 \mathrm{~m}$. No presente caso, utilizando-se de um sistema RTK com acurácia indicada da ordem de $0,025 \mathrm{~m}$, e utilizando a configuração recomendada pelo fabricante, não se conseguiu controle eficaz das faixas de sobreposição, resultando em até $9 \mathrm{~m}$ de sobreposição, ou abertura prematura da pulverização. Isto significa que o pulverizador pode não estar respondendo aos requisitos mínimos para o perfeito funcionamento do controlador.

\section{CONCLUSÕES}

A metodologia proposta para avaliar o controlador automático de seções e pulverização possibilitou a determinação dos tempos e distâncias de início ou término da pulverização. Foi possível determinar interações significativas entre os diferentes sinais de GPS. Embora houvesse casos em que determinado sinal de GPS se destacou em relação aos demais, não se pode concluir qual foi o melhor de forma generalizada. Conclui-se que outros fatores estão contribuindo para a diluição do erro de posicionamento do GPS.

A configuração recomendada pelo fabricante e adotada para a avaliação do controlador automático de seções e pulverização não atende a todas as situações de trabalho, sendo necessária para cada velocidade uma configuração específica a fim de se reduzirem os desvios no ponto de abertura e diminuição das sobreposições excessivas.

\section{AGRADECIMENTOS}

À empresa Máquinas Agrícolas Jacto S.A., nas pessoas do Fábio Pernassi Torres, João Batista Kimura Filho, Fernando Delarco e Wilson Marcos Stanislavski; à empresa Santiago \& Cintra, nas pessoas do Rodrigo Tamani e Rinaldo Lorenzoni.

\section{REFERÊNCIAS}

BATTE, M.T.; EHSANI, M.R. The economics of precision guidance with auto-boom control for farmer-owned agricultural sprayers. Computers and Electronics in Agriculture, New York, v. 53, p.28-44, 2006.

BOSSU, J.; GÉE, C.; TRUCHETET, F. Development of a machine vision system for a real time precision sprayer. Electronic Letters on Computer Vision and Image Analysis, Barcelona, v.7, n.3, p.54-66, 2008.

LIMA, T.C.B.; UMEZU, C.K.; CAPPELLI, N.L.; NUNES, E.F. Equipamento microprocessado para geração de sinal de correção diferencial, em tempo real, para GPS. Engenharia Agrícola, Jaboticabal, v.26, n.2, p.537-545, 2006. Disponível em: < http://www.scielo.br/pdf/eagri /v26n2/23.pdf> Acesso em: 21 jul. 2009.

McBRATNEY, A.; BRETT, W.; TIHOMIR, A.; JOHAN, B. Future Directions of Precision Agriculture. Precision Agriculture, New York, v.6, n.1, p.7-23, 2005.

SCHULLER, J.K. Design for dynamic response of sprayer-applicators. Journal of Fertilizer Issues, Manchester, v.8, n.3, p.69-73, 1991.

STAFFORD, J.V.; AMBLER, B.; LARK, R.M.; CATT, J.A. Mapping and interpreting the yield variation in cereal crops. Computers and Electronics in Agriculture, New York, v.14, p.101-119, 1996.

ZAMBOLIM, L.; CONCEIÇÃO, M.Z. da; SANTIAGO, T. O que os engenheiros agrônomos devem saber para orientar o uso de produtos fitossanitários. 3.ed. Viçosa: UFV/DFP, 2008. 464 p. 